\title{
Capacité des spermatozoïdes à se fixer sur la zone pellucide
}

\author{
S. HamamaH, M. JeaN*, D. Royère, P. BarRiÈRE*
}

Unité de Biologie de la Reproduction, Département de Gynéco-Obs, Reproduction humaine, et Médecine foetale, Hôpital Bretonneau, 37044 Tours ; * Laboratoire de Biologie de la

Reproduction, Département de Gynéco-Obs, Hôpital Mère-Enfant, 44035 Nantes

\section{RESUME}

Le spermatozoïde après sa libération de l'épithélium séminifère subit dans l'épididyme une série de modifications fonctionnelles et métaboliques lui conférant la capacité d'assurer la fécondation. La fécondation est le processus essentiel de la reproduction sexuée, elle permet d'initier la formation d'un nouvel être par la fusion de deux cellules germinales : le gamète mâle (spermatozoïde) et le gamète femelle (ovocyte).

Pour que la fécondation ait lieu, il faut que les spermatozoïdes reconnaissent la zone pellucide (ZP), s'y fixent, qu'ils la pénètrent et qu'il y ait fusion avec la membrane plasmique de l'ovocyte.

L'affinité des spermatozoïdes à reconnaître et à se fixer -sur la zone pellucide (ZP) apparaît au cours du transit dans l'épididyme, et elle est parallèle à l'acquisition du pouvoir fécondant, et contrôlée par les androgènes

Deux modalités d'interactions entre les spermatozoïdes et la ZP ont pu être observées : a) chez la plupart des mammifères, la fixation des spermatozoïdes n'a lieu que si la membrane plasmique est intacte, il en est de même chez l'homme, b) chez le hamster, la fixation du spermatozoïde sur la ZP ne peut s'effectuer qu'après la réaction acrosomique.
Mots clés : spermatozoïde, zone pellucide, interaction.

\section{INTRODUCTION}

Chez les Mammifères, la fécondation est le résultat d'une succession d'événements complexes qui assurent, grâce à la fusion de deux gamètes haploïdes (le spermatozoïde et l'ovocyte), la création d'un nouvel individu. Les étapes initiales de reconnaissance et d'interaction des gamètes sont capitales dans le déroulement de la fécondation. Le spermatozoïde doit d'abord reconnaître la zone pellucide de l'ovocyte, s'y fixer puis la traverser pour finalement venir fusionner avec la membrane plasmique de l'ovocyte. L'interaction entre les gamètes fait intervenir des molécules situées, d'une part sur la tête du spermatozoïde et, d'autre part, sur la zone pellucide de l'ovocyte.

La détermination de la nature et du rôle des molécules impliquées dans l'interaction spermatozoides-zone pellucide représente un des axes de recherche les plus importants dans le diagnostic de l'infécondité à l'échelle moléculaire.

1. La maturation épididymaire des spermatozoïdes

Au cours de la maturation épididymaire, le spermatozoïde subit des modifications qui se caractérisent par : 
- le développement d'une motilité progressive,

- l'acquisition d'une spécificité de reconnaissance et de fixation à l'ovocyte,

- l'acquisition du pouvoir fécondant, et

- la faculté à induire un développement embryonnaire normal.

Ces modifications des spermatozoïdes sont accompagnées de changements dans les caractéristiques de surface membranaire qui contribuent au remplacement ou au masquage des composés de haut poids moléculaire par d'autres de poids plus réduits [1]. Ces différentes protéines, pour la plupart sialylées ou glycosylées, sont sécrétées par l'épithélium épididymaire sous l'action des androgènes et se fixent à la membrane du spermatozoïde [2]. La fixation de ces protéines entraîne des modifications de la structure membranaire du spermatozoïde [3] en relation avec les interactions avec la ZP $[4,5]$.

\section{La capacitation}

La capacitation des spermatozoïdes est une étape indispensable à la fécondation chez tous les Mammifères [6]. Elle correspond aux modifications cellulaires et moléculaires subies par le spermatozoïde dans les voies génitales femelles qui le préparent à subir la réaction acrosomique (RA) [7].

D'une façon générale, la capacitation entraine des modifications importantes, parmi lesquelles :

a) Les modifications membranaires du spermatozoïde : pendant la maturation épididymaire et au cours de l'éjaculation, des protéines issues de l'épididyme et des glandes annexes se fixent au glycocalyx des spermatozoïdes et inhibent leur pouvoir fécondant en stabilisant la membrane [8]. $\mathrm{Au}$ cours de la capacitation, trois types de changements sont en cause selon la nature de la liaison de ces protéines avec la membrane plasmique :
- des protéines fixées au glycocalyx par des liaisons non covalentes sont relarguées dans les voies génitales féminines [9],

- des protéines de structure ou d'origine épididymaire incorporées dans la membrane subissent une perte de résidus glucidiques confirmée par la disparition progressive, au cours de la capacitation, de lectines fixées à la surface du spermatozoïde [10], et

- des protéines peuvent migrer et se redistribuer localement pour créer des zones fluides, pauvres en protéines, qui sont les futurs points de fusion, lors de la RA, entre la membrane plasmique et la membrane acrosomique externe [11]. Selon le modèle proposé par Davis et al. [12], la déstabilisation de la membrane du spermatozoïde serait liée à la diminution du rapport cholestérol / phospholipides associée à une augmentation de la perméabilité au $\mathrm{Ca}++$.

\section{b) Le changement de motilité}

Le phénomène de capacitation s'accompagne d'une transformation des paramètres du déplacement des spermatozoïdes en un mouvement non linéaire, caractérisé par un battement flagellaire de forme asymétrique et de forte amplitude. Ce mouvement particulier des spermatozoïdes capacités est appelé «hyperactivation» [7]. L'entrée de $\mathrm{Ca}++$ dans la cellule, liée à la déstabilisation de la membrane, permettrait l'activation d'une adénylcyclase-Ca++dépendante. L'augmentation du taux d'AMPc alors observée serait responsable de la phosphorylation de la tubuline et du changement de motilité [8].

Lors de leur transit dans les voies génitales féminines, la progression des spermatozoïdes est assurée par des battements flagellaires symétriques avec une fréquence importante et une faible amplitude. Lorsqu'ils parviennent au voisinage de l'ovocyte, les spermatozoïdes capacités présentent alors cette mobilité hyperactivée et faible- 
ment progressive. Chez l'Homme, à la différence d'autres Mammifères, la proportion de spermatozoïdes hyperactivés est faible et voisine de $20 \%$ [13].

Pour les spermatozoïdes fixés à la ZP, le battement flagellaire de forte amplitude contribue, pour une part, à la progression du spermatozoïde à travers cette enveloppe.

\section{Les sites d'affinité de la zone pelluci- de pour les spermatozoïdes}

La zone pellucide, enveloppe acellulaire entourant l'ovocyte des Mammifères, est synthétisée au cours de la folliculogénèse et assure la protection de l'ovocyte puis de l'embryon jusqu'au stade pré-implantatoire [14]. Elle est impliquée dans l'interaction gamètique en assurant la reconnaissance homospécifique et la fixation des spermatozoïdes ainsi que le déclenchement de la réaction acrosomique.

Les études réalisées chez la Souris [15] ont fourni l'essentiel des informations disponibles actuellement sur la composition biochimique de la zone pellucide. Elle possède une structure tridimensionnelle formée de 3 glycoprotéines majeures appelées ZP1, ZP2 et ZP3.

ZP2 et ZP3 sont associées en alternance et forment des filaments de longueur (2-3 $\mathrm{mm}$ ) et d'épaisseur (7-18 $\mathrm{nm}$ ) uniformes. Ces filaments sont reliés entre eux par ZP1.
La glycoprotéine ZP3 est constituée d'une composante polypeptidique sur laquelle sont greffées des résidus oligosaccharidiques (fucose et $\mathrm{N}$-acétyl-glucosamine notamment).

Les 3 glycoprotéines de la zone pellucide (ZP1, ZP2 et ZP3), classées par ordre décroissant de poids moléculaire, sont retrouvées dans de nombreuses espèces (Tableau 1). Une similitude de fonction pour les glycoprotéines de même nom reste cependant à démontrer. D'une façon générale, cette architecture moléculaire confère à la zone pellucide une structure élastique, perméable aux enzymes, aux immunoglobulines et aux virus de petite taille.

La structure de la zone pellucide humaine, désormais bien établie [16, 17], est comparable à celle des autres Mammifères. Elle est formée des 3 glycoprotéines majeures ZP1, ZP2 et ZP3 avec des poids moléculaires apparents respectifs de 97,82 et $70 \mathrm{kD}$.

Linteraction spermatozoide-zone pellucide, à l'image des interactions entre cellules somatiques, serait du type ligand-récepteur [18]. Selon le modèle proposé chez la Souris [19], l'interaction du spermatozoïde avec la zone pellucide peut être séparée en 2 étapes :

- la première étape débute par une liaison labile et réversible du spermatozoïde avec la zone pellucide,

Tableau 1 : Glycoproteines de la zone pellucide dans diverses espèces animales et chez l'homme.

\begin{tabular}{lcccll}
\hline Espèces & $\begin{array}{l}\text { ZP 1 } \\
\text { (kD) }\end{array}$ & $\begin{array}{c}\text { ZP2 } \\
\text { (kD) }\end{array}$ & $\begin{array}{c}\text { ZP3 } \\
\text { (kD) }\end{array}$ & $\begin{array}{l}\text { ZP4 } \\
(\mathbf{k D})\end{array}$ & Reférences \\
\hline Vache & $94-110$ & $82-94$ & $70-76$ & & Florman \& First, 1988 \\
Chien & $90-100$ & $70-95$ & $50-100$ & & Maresh \& Dunbar, 1987 \\
Hamster & 240 & 150 & 80 & & Ahuja \& Bolwel, 1983 \\
Cheval & $93-120$ & $73-90$ & $45-80$ & & Miller et al, 1992 \\
Souris & 200 & 120 & 83 & & Bleil \& Wassarman, 1980 \\
Lapin & $100-132$ & $81-100$ & $68-125$ & & Dunbar et al, 1981 \\
Porc & $80-90$ & $60-65$ & $55-60$ & $20-25$ & Hedrick \& Wardrip, 1986 \\
Homme & $90-110$ & $64-78$ & $57-73$ & & Shabanowitz \& O'Rand, 1988 \\
\hline
\end{tabular}


- puis, l'interaction des chaînes oligosaccharidiques de ZP3 avec un (ou plusieurs) récepteur de la membrane plasmique du spermatozoïde établit une liaison irréversible et spécifique entre les gamètes.

Cette fixation primaire est à l'origine du déclenchement de la réaction acrosomique (RA) [20].

Lorsque cette réaction s'est produite, l'interaction d'un récepteur situé sur la membrane interne de l'acrosome (ou dans la matrice acrosomique) avec un second ligand de la zone pellucide (ZP2) devient possible : c'est l'étape de fixation secondaire [21].

Si chez la Souris, ZP3 est le seul composant de la zone pellucide capable d'agir comme site de reconnaissance initiale [22], les principaux groupes de glycoprotéines de la zone pellucide humaine auraient tous le rôle de ligands pour les protéines du spermatozoïde humain [17] et pourraient tous déclencher la réaction acrosomique [22].

\section{Les récepteurs du spermatozoïde pour la zone pellucide}

Si la nature et la localisation des récepteurs spermatiques impliqués dans l'interaction avec la zone pellucide ne sont pas encore totalement précisées, le rôle des protéines de la membrane plasmique du spermatozoïde a cependant pu être établi dans plusieurs espèces (Tableau 2).

Chez la Souris, une galactosyltransférase portée par la membrane plasmique du spermatozoïde pourrait être impliquée dans la reconnaissance et la fixation des spermatozoïdes [23, 24]. La liaison avec la zone pellucide résulterait donc d'un pontage entre la membrane plasmique du spermatozoïde et un résidu $\mathrm{N}$-acétylglucosamine de la ZP3 par l'intermédiaire de cette enzyme.

Chez l'Homme, il n'y aurait pas de galactosyltransférase mais, une alpha-D-mannosidase, retrouvée à la surface du spermatozoïde serait le récepteur pour ZP3 [25].
Grâce à des anticorps anti-phosphotyrosine (anti P-Tyr), des protéines servant de substrat à des protéines-kinases ont également pu être identifiées dans les spermatozoïdes murins [26] et humains [27]. Après capacitation, $15 \%$ des spermatozoïdes murins et $50 \%$ des spermatozoïdes humains possèdent des résidus P-Tyr au niveau de la région acrosomique. Chez la Souris, l'incubation de spermatozoïdes capacités avec des pellucides dissoutes fait apparaître une réactivité importante à l'anticorps anti P-Tyr pour une protéine de $95 \mathrm{kD}$, appelée P95 [26]. Chez l'Homme, la phosphorylation sur tyrosine d'une protéine de $94 \mathrm{kD}$ est également stimulée par l'exposition à des pellucides dissoutes [27]. Ainsi, à l'image de la Souris où la P95 se fixe à ZP3 [26], la P94 du spermatozoïde humain pourrait être impliquée dans l'interaction avec la zone pellucide [27]. L'analogie retrouvée entre 3 protéines du spermatozoïde humain $(16,18$ et $19 \mathrm{kD})$ et la RSA («Rabbit Sperm Antigen») qui interagit de façon spécifique avec la zone pellucide, est également en faveur du rôle de ces protéines comme récepteurs pour la zone pellucide chez l'Homme [28].

Une fois fixé à la zone pellucide (fixation primaire), le spermatozoïde effectue sa réaction acrosomique. Ce processus d'exocytose assure, d'une part, la libération du contenu acrosomique et, d'autre part, l'externalisation de la membrane interne de l'acrosome [29].

L'acrosine est une protéase localisée dans l'acrosome des spermatozoïdes de Mammiferres sous sa forme inactive, la proacrosine. Cette enzyme pourrait être impliquée dans la fixation secondaire qui intervient entre le spermatozoïde en cours de réaction acrosomique et les chaînes saccharidiques de ZP2 [30]. Les mouvements flagellaires associés à l'hydrolyse sélective de ZP1 par l'acrosine libérée lors de la réaction acrosomique assurent la progression du spermatozoïde à travers la zone pellucide [31]. 
Tableau 2 : Protéines et glycoprotéines de la membrane plasmique de spermatozoïdes impliquées dans la fixation sur la ZP.

\begin{tabular}{|c|c|c|c|c|c|}
\hline \multirow[t]{2}{*}{ Espèces } & \multicolumn{4}{|c|}{ Protéines et Glycoprotéines de spermatozoïdes $(\mathrm{kD})$} & \multirow[t]{2}{*}{ Références } \\
\hline & $\mathbf{I}$ & II & III & IV & \\
\hline Souris & 95 & $\begin{array}{c}45-54 \\
56\end{array}$ & 33 & $\begin{array}{c}15 \\
16-19\end{array}$ & $\begin{array}{l}\text { Leyton et al, } 1989 \\
\text { Lopez et al, } 1985 \\
\text { Bleil et al, } 1990 \\
\text { Poirier et al, } 1986 \\
\text { O'Rand et al, } 1985\end{array}$ \\
\hline Porc & $\begin{array}{c}70 \\
63-88 \\
67\end{array}$ & $\begin{array}{c}45 \\
45-53 \\
53\end{array}$ & $\begin{array}{l}30 \\
38\end{array}$ & $\begin{array}{l}<20 \\
16-18 \\
18\end{array}$ & $\begin{array}{l}\text { Peterson et al, } 1985 \\
\text { O'Rand et al, } 1985 \\
\text { Brown et al, } 1985\end{array}$ \\
\hline Lapin & 64 & 54 & 32 & $14-18$ & O'Rand et al, 1985 \\
\hline Homme & 60 & & 35 & $16-19$ & $\begin{array}{l}\text { O'Rand et al, } 1990 \\
\text { Naz et al, } 1991\end{array}$ \\
\hline
\end{tabular}

Chez la Souris, après l'étape de fixation primaire du spermatozoïde, ZP3 est inactivée en ZP3f [15] perdant ainsi sa capacité à fixer de nouveaux spermatozoïdes. Après la fixation secondaire, la protéolyse de ZP2 qui empêche la pénétration de spermatozoïdes surnuméraires [30] complète le processus de blocage de la polyspermie. Ces modifications de ZP2 et ZP3, observées au cours de la fécondation de l'ovocyte de Souris, sont provoquées par la libération dans l'espace périvitellin des enzymes des granules corticaux [32]. Chez l'Homme, le blocage de la polyspermie pourrait être associé à une dégradation de ZP1 [17].

\section{La réaction acrosomique}

Chez les Mammifères, la réaction acrosomique (RA) est caractérisée par la fusion et la vésiculisation des membranes plasmique et acrosomique externe (Figure 1). Ce processus d'exocytose a deux conséquences :

- il assure la libération du contenu acrosomique : la hyaluronidase et l'acrosine, deux enzymes protéolytiques présentes dans l'acrosome, en digérant localement les glycoprotéines de la zone pellucide, favorisent la progression du spermatozoïde à travers cette structure ;

- il permet l'externalisation de la membrane interne de l'acrosome : le système proacrosine-acrosine lié à cette membrane interne peut alors interagir avec ZP2 et établir ainsi la fixation secondaire du spermatozoïde avec la zone pellucide.

\section{a) Déclenchement de la réaction acro- somique}

Chez l'Homme, dans un milieu capacitant, 10 à $20 \%$ des spermatozoïdes peuvent subir spontanément la RA [33]. Même si ces spermatozoïdes peuvent se fixer à la $\mathrm{ZP}$, il apparait cependant que l'achèvement de la RA à distance de l'ovocyte rend le spermatozoïde incapable de pénétrer la zone pellucide [34].

Chez de nombreux Mammifères dont l'Homme, la RA peut être déclenchée par l'incubation des spermatozoïdes avec des zones pellucides homologues dissoutes. 
Chez la Souris, en plus de son rôle de récepteur pour le spermatozoïde, ZP3 intervient dans le déclenchement de la RA. Le squelette peptidique de cette glycoprotéine est responsable de l'induction de la RA alors que les résidus saccharidiques de ZP3 sont impliqués dans la fixation primaire du spermatozoïde à la zone pellucide. Selon Leyton et al. [35], ZP3 en se fixant à la P95 du spermatozoïde entraînerait une agrégation des récepteurs dans la membrane du gamète mâle. Cette agrégation stimulerait l'activité d'une protéine-kinase intrinsèque, provoquant l'autophosphorylation du récepteur et le déclenchement de la cascade d'événements cellulaires et moléculaires de la RA.

Chez l'Homme, cette question reste controversée malgré plusieurs travaux en faveur du rôle de ZP3 dans l'induction de la RA [36] notamment en raison du fort degré d'homologie entre les ARN messagers humain et murin de ZP3 [37].

\section{b) Aspects moléculaires de la réaction acrosomique}

L' augmentation de la perméabilité au $\mathrm{Ca}++$ de la membrane plasmique, conséquence de la capacitation, constitue le signal de départ de la RA. L'entrée du Ca++ dans le spermatozoïde a plusieurs conséquences qui vont aboutir à l'exocytose du contenu acrosomique :

- le Ca++ stimule un système adénylcyclase/AMPc. L'AMPc qui joue le rôle de second messager active des protéineskinases et provoque la libération de stocks de $\mathrm{Ca}++$ intracellulaire.

- le Ca++ inhibe des ATPases membranaires entrainant un afflux de protons et une augmentation du pH de l'acrosome. Cette alcalinisation du contenu acrosomique pourrait stimuler la transformation de proacrosine en acrosine.

- le Ca++ stimule une activité phospholipase A2 par l'intermédiaire de l'acrosine. La phospholipase A2 transforme les phospholipides membranaires en acides gras insaturés et en lysophospholipides. La lysophosphatidylcholine et la lysophosphatidyléthanolamine ainsi produites auraient une action sur la fusion des membranes plasmique et acrosomique externe. L'albumine sérique pourrait éliminer les acides gras formés pour éviter l'inhibition de la phospholipase A2 ou jouer un rôle de chélateur pour le cholestérol. En diminuant ainsi le rapport cholestérol / phospholipides, l'albumine sérique participerait à la déstabilisation membranaire et, à terme, à la RA.

\section{CONCLUSION}

Du fait des rôles majeurs de la ZP dans le processus de la fécondation:

- interaction avec les spermatozoïdes,

- induction de la raction acrosomique et

- le contrôle de la polyspermie, la ZP est le cible attractive pour le développement d'un vaccin contraceptif.

Des analyses immunologiques ont révélé la présence au sein de la ZP d'antigènes spécifiques d'espèce impliqués dans la reconnaissance de spermatozoïdes pour la ZP, ayant une réaction croisée avec d'autres espéces de mammiféres.

\section{REFERENCES}

1. Dacheux JL, Dacheux F. : Maturation membranaire des spermatozoïdes épididymaires. Contracept Fertil Sex $1990 ; 18: 538-540$.

2. Kohane AC, Gonzales-Echeverrla FM, Pineiro L, BLAQUIER JA. : Interaction of proteins of epididymal origin with spermatozoa. Biol Reprod 1980 ; $23: 737-744$.

3. Fraser LR. : Mechanisms controlling mammalian fertilization. In : Oxford reviews of reproductive biology. JR Clarke ed., Clanedon Press, Oxford, 1984, 174-195.

4. Gonzalez-Echeverria F., Cuasnicu P., Plazza A, Pineiro L, Blaquier J. : Addition of an androgenfree epididymal protein extract increases the ability of immature hamster spermatozoa to fertilize in vivo and in vitro. $\mathrm{J}$ Reprod Fertil $1984 ; 71: 433-437$. 
5. MoORe H., Hartman T. : In vitro development of the fertilizing ability of hamster epididymal spermatozoa after co-culture with epithelium from the proximal cauda epididymis. J Reprod Fertil 1986 ; $78: 347-352$.

6. AUstin CR. : Capacitation of spermatozoa. Int $\mathrm{J}$ Fertil 1967; $12: 25-31$.

7. YANAGIMACHI R. : Mechanisms of fertilization in mammals. In : Fertilization and embryonic development in vitro. Mastroianni L, Biggers JD eds, New-York, Plenum Press, 1981, 81-187.

8. SidHU KS, GURAYA SS. : Cellular and molecular biology of capacitation and acrosome reaction in mammalian spermatozoa. Int Rev Cytol 1989; 118 :231-280.

9. Villarroya S., Scholler R. : Lateral diffusion of a human sperm-head antigen during incubation in a capacitation medium and the induction of acrosome reaction in vitro. J Reprod Fertil $1987 ; 80$ : 545-562.

10. KoEHLER JK. : Lectins as probes of the spermatozoon surface. Arch Androl $1981 ; 6$ : 197-217.

11. SuzuKi F., ANAGIMACHI R. : Changes in the distribution of intra-membraneous particles and filipin-reactive membrane sterols during in vitro capacitation of golden hamster. Garnetc Rcs 1989 ; $23: 335-347$.

12. Davis BK, Byrne R., Hungund B. : Studies on the mechanism of capacitation. II. Evidence for lipid transfer between plasma membrane of rat sperm and serum albumin during capacitation in vitro. Biochim Biophys Acta 1979 ; 558 : 257-266.

13. Robertson L, WOLF DP, TASH JS. : Temporal changes in motility parameters related to acrosomal status : identification and characterization of populations of hyperactivated human sperm. Biol Reprod $1988 ; 39: 797-805$.

14. DieTL J. : Ultrastuctural aspects of the developing mammalian zona pellucida. In Dietl J editor: The mammalian egg coat : structure and function. Springer-Verlag, Berlin, 1989, 49-60.

15. BLEIL JD, WASSARMAN PM. : Structure and function of the zona pellucida : identification and characterization of the proteins of the mouse oocyte's zona pellucida. Dev Biol 1980a ; 76 : 185-203.

16. Shabanowitz RB, O'RAND MG. : Characterization of the human zona pellucida from fertilized and unfertilized eggs. J Reprod Fertil 1988a ; 82 : 151-161.

17. SHABANowitz RB, O'RAND MG. : Molecular changes in the human zona pellucida associated with fertilization and human sperm-zona interactions. Ann N Acad Sci 1988b ; 541 : 621-632.
18. KoPF GS, WILDE MW. : Signal transduction processes leading to acrosomal exocytosis in Mammalian spermatozoa. TEM September/October. Elsevier, Science, Publishing Co, 1990, 362-368.

19. Bleil JD, Wassarman PM. : Galactose at the nonreducing terminus of $\mathrm{O}$-linked oligosaccharides of mouse egg zona pellucida glycoprotein ZP3 is essential for the glycoprotein's sperm receptor activity. Proc Natl Acad Sci USA 1988a ; 85 : 6778-6782.

20. SALING PM. : Mammalian sperm interaction with extracellular matrices of the egg. Oxford Rev Reprod Biol $1989 ; 11: 339-388$.

21. BLEIL JD, WASSARMAN PM. : Identification of a secondary sperm receptor in the mouse egg zona pellucida : role in maitenance of binding of acrosome-reacted sperm to egg. Dev Biol 1988b ; 126 : 376-385.

22. Wassarman PM, Bleil JD, Florman hM, Greve JM, ROLLER RJ, SAlzMANN GS. : Nature of the mouse egg's receptor for sperm. In: Hedrick JL ed, The molevular and cellular biology of fertilization, New-York, Plenum Press, 1986, 55-77.

23. Lopez LC, Bayna EM, Litoff D, Shaper NL, SHaPER JH, SHUR BD. : Receptor function of mouse sperm surface galactosyl-transferase during fertilization. J Cell Biol 1985; 101 : 1501-1510.

24. MACeK MB, Shur BD. : Protein carbohydrate complementary in mammalian gamete recognition. Gamete Res $1988 ; 20: 93-109$.

25. Tulsiani DRP, SkUdLaRek MD, ORGEbin-CRIST MC. : Human sperm plasma membrane possess alpha-D-mannosidase activity but no galactosyltransferase activity. Biol Reprod $1990 ; 42$ : 843-858.

26. LeYton L, SALING PM. : $95 \mathrm{kD}$ sperm proteins bind ZP3 and serve as tyrosine kinase substrates in response to zona binding. Cell $1989 ; 57: 1123-1130$.

27. NAZ RK, AHMAD K., KUMAR R. : Role of membrane phosphotyrosine proteins in human spermatozoal function. J Cell Sci $1991 ; 99: 157-163$.

28. O'Rand MG, Widgreen EE, Nikolajczyk BS, RICHARDSON RT, SHABANOwitz RB. : Receptors for zona pellucida on human spermatozoa. In : Gamete interaction: Prospects for immunocontraception, Wiley-Liss Inc, 1990, 213-224.

29. YANAGIMACHI R. : Mammalian fertilization. In: The physiology of reproduction. Knobil E. Neill J eds, New-York, Raven Press Ltd, 1988.

30. BLeIL JD, WASSARMAN PM. : Identification of a secondary sperm receptor in the mouse egg zona pellucida: role in maitenance of binding of acrosome-reacted sperm to egg. Dev Biol 1988b ; 126 : 376-385. 
31. Dunbar BS, Dudkiewicz AB, Bundman DS. : Proteolysis of specific porcine zona pellucida glycoproteins by boar acrosin. Biol Reprod 1985 ; 32 : 618-630.

32. WolF DP, LANZENDORF SE. : Fertilization in man. In : A comparative overview of mammalian fertilization. Dunbar BS, O'Rand MG eds, Plenum Press, New-York, 1991, 385-400.

33. Wolf DP, BoldT J. BYRD W. BEchTol KB. : Acrosomal status evaluation in human ejaculated sperm with monoclonal antibodies. Biol Reprod 1985 ; 32 : 1157- 1162 .

34. TESARIK J. : Reconnaissance gamétique et interaction du sperme avec la zone pellucide. Contracept Fertil Sex $1990 ; 18: 546-548$.

35. Leyton L, Bunch D, Le Guen P. Selub M, Saling P. : Regulation of acrosomal exocytosis by sperm binding to the zona pellucida. In: Gamete interaction : Prospects for immunocontraception, Wiley-Liss Inc, 1990, 225-238.

36. TesariK J., TestaRT J. : Human sperm-egg interactions and their disorders: implications in the management of infertility. Human Reprod 1989 ; $4: 729-741$.

37. Chamberlain ME, Dean J. : Human homolog of the mouse sperm receptor. Proc Natl Acad Sci USA $1990 ; 87: 6014-6018$.

\section{ABSTRACT \\ Sperm-Zona pellucida interactions \\ S. Hamamah, M. Jean, D. RoyeRe, P. Barriere \\ After liberation from the seminiferous epithelium, the spermatozoa (SPZ), undergo in the epididymis a serie of functional and metabolic modifica- tions resulting the capacity to ensure fertilization. Fertilization is the funda- mental process in sexual reproduction}

as it permits the initiation and the formation of a new being by the fusion of two germinal cells : the male gamete (spermatozoa) and the female gamete (oocyte). For fertilization to occur the SPZ must recognize the zona pellucida (ZP), bind to it, penetrate it and fuse with the oocyte plasma membrane.

Sperm binding to the $\mathrm{ZP}$ is an early, crucial event leading to fertilization and pre-embryo development. In mammals, sperm-ZP binding follows a serie of steps that occur in a well-defined chronological order : a) A loose association between $S P Z$ and $Z P$ referred to as "attachment". This shortlived interaction is heterospecific. b) Attachment is followed by a more distinct and persistent association of SPZ with ZP, thus called "binding". This sperm-zona interaction is species-specific, irreversible and mediated by complementary receptors present on the SPZ head and the ZP. c) The bound SPZ then undergoes the acrosome reaction (AR). Which involves fusion and vesiculation of the SPZ outer acrosomal membrane and plasma membrane leading to the release of acrosomal contents and the exposure of the inner acrosomal membrane. This AR is essential for SPZ passage through the $\mathrm{ZP}$ and to access to the oocyte plasma membrane where gamete fusion occurs.

Key words : zona pellucida, sperm receptor. 